\title{
Exposing Image Forgery through Contrast Enhancement
}

\author{
Priya Ann Koshy ${ }^{1}$, Kavitha N. Nair ${ }^{2}$ \\ ${ }^{1}$ Dept. of E.C.E, Mahatma Gandhi University College of Engineering, Thodupuzha, Kerala, India \\ ${ }^{2}$ Assistant Professor, Dept. of E.C.E., Mahatma Gandhi University College of Engineering, Thodupuzha, Kerala
}

\begin{abstract}
Today digital images may be used for several beneficial purposes such as evidence in court. Image forgeries may happen, so verification is needed to detect the authenticity of the image. Contrast enhancement is used for adjusting the brightness and contrast of a digital image. Malicious users may also perform contrast enhancement locally for creating a realistic composite image. Here proposes two algorithms, first for detecting the contrast enhancement based manipulation involved in JPEG compressed images and the second one is used for detecting composite image. First, we focus on the detection of global contrast enhancement applied to the previously JPEG-compressed images, which are widespread in real applications. The histogram peak/gap artifacts incurred by the JPEG compression and pixel value mappings are analyzed theoretically, and Receiver Operating Characteristic (ROC) curves are generated for evaluation. Second, we propose to identify the composite image created by enforcing contrast adjustment on either one or both source regions. The positions of detected blockwise peak/gap bins are clustered for recognizing the contrast enhancement mappings applied to different source regions.
\end{abstract}

Keywords: digital forensics, image forgery, contrast enhancement, composite image.

\section{Introduction}

The immediate development in the field of digital image editing, image manipulation is becoming very easy and convenient. At times it is legal and beneficial and sometimes become suspicious. In some applications, such as a crime related situation it is necessary to detect any suspicious activity have occurred and history of that image. Contrast enhancement is used to adjust the brightness and contrast of digital images. Malicious users may perform contrast enhancement locally for creating realistic composite images.

As such it is significant to detect contrast enhancement blindly for verifying the originality and authenticity of digital images. Some forensic methods detects whether manipulation occurred but fails to detect which specific type of activity is done. Another category focus on detecting specific image manipulation. The works focus on detecting the different types of alterations, which can be divided into two categories: 1) non-content-changing operations including resampling, compression, sharpening filtering, contrast enhancement 2) content-changing operations, like composition and splicing. The contrast enhancement forensic algorithms performs well under the assumption that the gray level histogram of an unchanged image will have a smooth curve. But it is well-known that low quality factor (Q) generates blocking artifacts, which cause unsmoothness histogram. To solve such a problem, we propose to detect the global contrast enhancement not only in uncompressed or high quality JPEG images but also in low quality ones.

The remaining part of thesis is organized as follows Section II gives an overview of related work in digital image enhancement is briefly reviewed. Section III deals with the research work and its implications. Section IV shows the experiment results of each stage is presented. Section V deals with conclusion and potential future work is discussed.

\section{Related Works}

Contrast enhancement is a pixel value mapping, $y=m(x)$, where $m(\cdot)$ is the mapping function, $x, y=0,1,2, \ldots, 255$ are the pixel gray level before and after mapping. Here 8-bit grayscale images are considered. It is shown that the histogram of unaltered images typically conforms to a smooth envelope, while that of enhanced images presents peak/gap artifacts. Based on such observation, proposed a contrast enhancement detection algorithm as follows:

1) Get the image's gray level histogram $h(x)$.

2) Perform $g(x)=p(x) h(x)$, where $p(x)$ is a pinch-off function.

3 ) The high frequency energy metric $F$ as

$$
F=\frac{1}{N} \sum_{W}|b(w) G(w)|
$$

$G(w)$ is the discrete Fourier transform of $g(x) \cdot b(w)=1$, if $|w|$ $\geq c ; b(w)=0$, otherwise, where $c$ is a cutoff frequency. $N$ is the number of pixels.

4) If value of $F$ is greater than the decision threshold, contrast enhancement is detected.

\section{Proposed Method}

Here proposes an efficient algorithm which detects the enhancement in both uncompressed and previously JPEGcompressed images. The proposed system contains two different methodologies :

\subsection{Peak/ Gap Artifacts Incurred By JPEG Compression}

JPEG compression of an 8-bit gray-scale image includes the steps: pixel value shift from the range $[0,255]$ to $[-128$, 127], $8 \times 8$ blocks division, block discrete cosine transform (DCT), quantization and entropy encoding. On applying DCT, DC coefficient is large while the others are small in flat

\section{Volume 4 Issue 12, December 2015}




\section{International Journal of Science and Research (IJSR) \\ ISSN (Online): 2319-7064 \\ Index Copernicus Value (2013): 6.14 | Impact Factor (2014): 5.611}

blocks. It is assumed that there exist blocks in which the quantized DC coefficient is non-zero. After inverse DCT, the decompressed flat block denoted as $\mathrm{I}_{\mathrm{b}}$ as in (2)

$$
\mathrm{I}_{\mathrm{b}}(\mathrm{i}, \mathrm{j})=\text { round }\left(\mathrm{qn}_{\mathrm{d}} / 8+128\right)
$$

Here, $\mathrm{i}, \mathrm{j}=0,1,2, \ldots, 7$ are local pixel coordinates. Round $(\cdot)$ is the rounding operator. $\mathrm{q}$ is DC quantization step. $\mathrm{n}_{\mathrm{d}}=\mathrm{L}$, $\mathrm{L}+1, \mathrm{~L}+2, \ldots, \mathrm{U}$ denotes the quantized DC coefficient, where $\mathrm{L}=\operatorname{round}(-128 \times 8 / \mathrm{q})$ and $\mathrm{U}=\operatorname{round}(127 \times 8 / \mathrm{q})$ are deduced from the definitions in JPEG compression standard. Let $c=q / 8=T 1 / T 2, T 1, T 2 \in Z$ are prime. $I_{b}(i, j)$ via $n_{d}$ is a scale round mapping. $\Psi_{c}(b)=\left\{n_{d} \mid b=\operatorname{round}\left(\mathrm{cn}_{\mathrm{d}}\right)\right\}$ set of $\mathrm{n}_{\mathrm{d}}$ mapped to the same value of $b$. Because of such periodic and non constant properties, candidate peaks may appear in the gray level histogram bin at k, where k denoted by (3)

$$
\mathrm{k} \in\left\{\mathrm{b}^{\prime} \mid \mathrm{b}^{\prime}=\operatorname{argmax}\left(\left|\Psi_{\mathrm{c}}(\mathrm{b})\right|\right)\right\}
$$

Two factors affect the de facto presence of histogram peak bins in a JPEG image: 1) the flatness; 2) JPEG quality factor. The larger flat regions and larger DC quantization step would cause more apparent peak bins. Strength of the histogram peak/gap artifacts incurred by JPEG compression can also be measured by the metric $\mathrm{F}$ defined as in (1)

Fig. 1 shows the cumulative distribution of $\mathrm{F}$ computed from UCID images and their compressed versions. It demonstrates that $\mathrm{F}$ of more than $85 \%$ uncompressed images lie below 0.06. After compression, $F$ values of the images increase and most of them become larger than 0.06. Moreover, the smaller the $\mathrm{Q}$ is, the larger the $\mathrm{F}$ becomes. Such results validate that peak/gap artifacts really exist in most middle/low quality JPEG images.

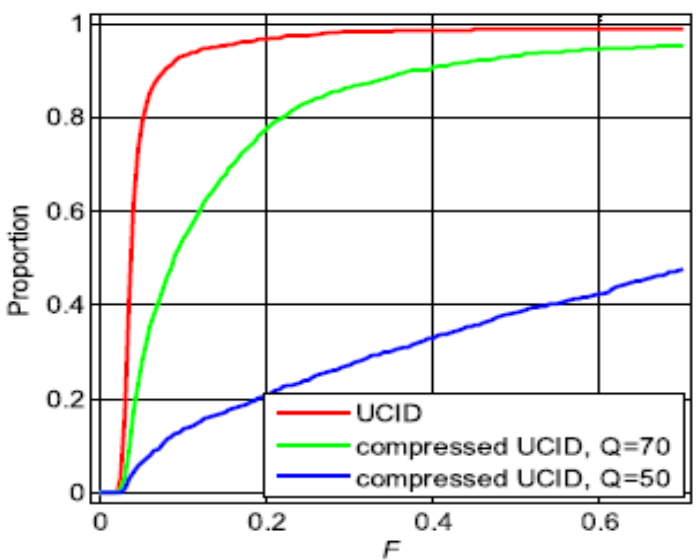

Figure 1: Cumulative distribution of $F$ values computed from UCID images and their JPEG versions

\subsection{Peak/Gap Artifacts Incurred By Contrast Enhancement}

The histogram of an enhanced image, $\mathrm{h}_{\mathrm{Y}}$, is written as

$$
\mathrm{h}_{\mathrm{Y}}(\mathrm{y})=\boldsymbol{\Sigma}_{\mathrm{x}} \mathrm{h}_{\mathrm{x}}(\mathrm{x}) \ell(\mathrm{m}(\mathrm{x})=\mathrm{y})
$$

where $\ell(\cdot)$ denotes the indicator function defined as $\ell(u)=1$, if $\mathrm{u}=1 ; \ell(\mathrm{u})=0$, otherwise. $\mathrm{h}_{\mathrm{x}}$ is the histogram of the original image. The equation shows that every value of $h_{y}$ must equal a single value of $h_{x}$, a sum of distinct $h_{x}$ values, or zero. Impulsive peaks will occur in $\mathrm{h}_{\mathrm{y}}$ at $\mathrm{y}$ values to which multiple $\mathrm{x}$ values are mapped, such as $\mathrm{y}=254$ in Fig. 2. Similarly, gaps will appear in $\mathrm{h}_{\mathrm{y}}$ at $\mathrm{y}$, for example, $\mathrm{y}=4$, to which no $\mathrm{x}$ values were mapped.

The gap bins with zero height, where no primary pixel values are mapped to, always appear in enhanced images. On the contrary, the zero height gap bins are absent in compressed images. The zero-height gap feature can be used to detect global contrast enhancement in both uncompressed and compressed images.

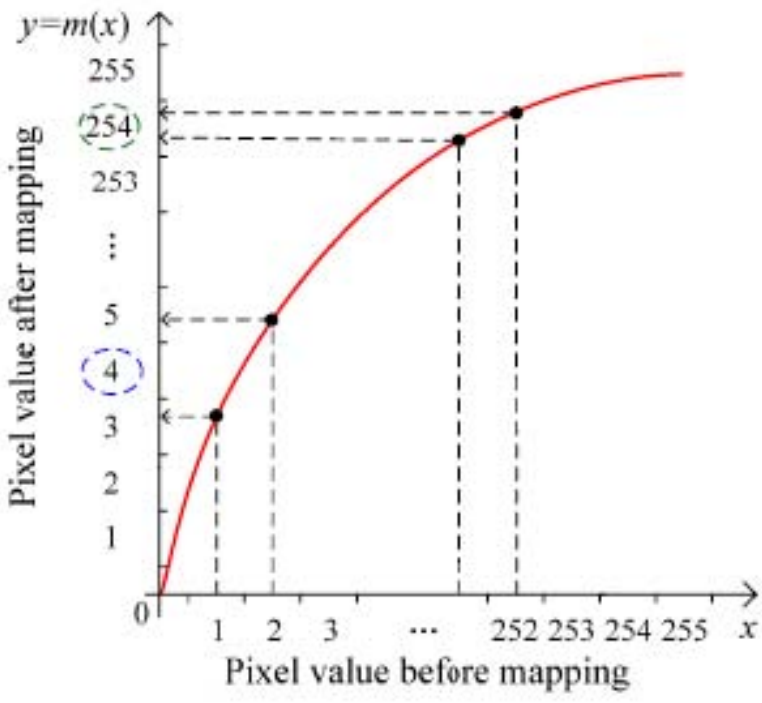

Figure 2: Peak/gap incurred by pixel value mapping

\subsection{Proposed Contrast Enhancement Detection Algorithm}

Here proposes the contrast enhancement detection algorithm: 1) Get image's normalized gray level histogram $h(x)$.

2) Find the bin at $k$ as a zero-height gap bin if it satisfies:

$$
\left\{\begin{array}{c}
h(k)=0 \\
\min \{h(k-1), h(k+1)\}>\tau \\
\frac{1}{2 w_{1}+1} \sum_{\mathrm{K}=\mathrm{k}-\mathrm{w}_{1}}^{\mathrm{k}+\mathrm{w}_{1}} \mathrm{~h}(\mathrm{x})>\tau
\end{array}\right.
$$

The first sub-equation shows that the current bin is null. To define a gap bin, the second sub-equation keeps two neighboring bins larger than the threshold $\tau$, as shown in Fig. 3. To exclude the zero-height gap bins, the average of neighboring $(2 \mathrm{w} 1+1)$ bins should be larger than $\tau$, as seen in the third sub equation. Here we focus on the detection of isolated zero-height gap bins.

3)Count the number of detected zero-height gap bins, denoted by $\mathrm{Ng}$. If it is larger than the decision threshold, contrast enhancement is detected, else not. 


\section{International Journal of Science and Research (IJSR) \\ ISSN (Online): 2319-7064}

Index Copernicus Value (2013): 6.14 | Impact Factor (2014): 5.611

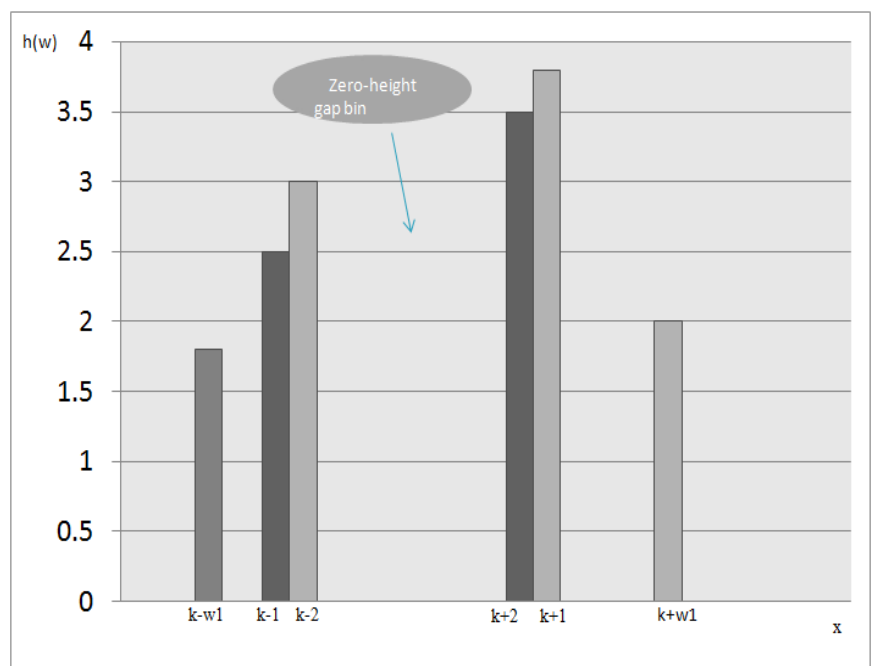

Figure 3: Definition of a zero-height gap bin at $\mathrm{k}$

\subsection{Identify Source-Enhanced Composite Images}

An algorithm is proposed to identify the source-enhanced composite image. The outline of the technique is shown in

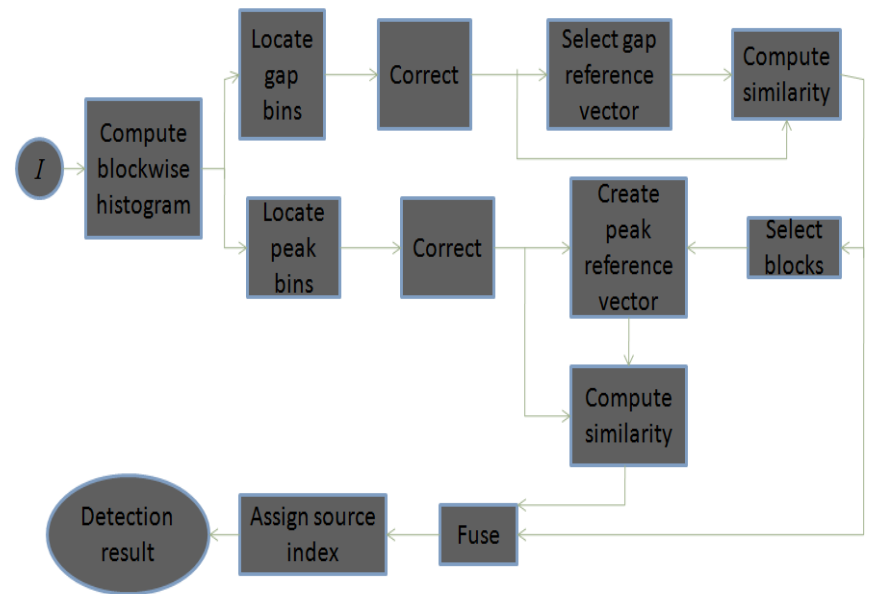

Figure 4: Flowchart of the proposed composite image detection technique

Fig. 4 Consistency between the peak/gap artifacts is checked for finding composite images.

\subsubsection{Blockwise Peak/Gap Bins Location}

To locate composition, the test image I is first divided into non-overlapping blocks. Here and below, $\mathrm{i}=1,2, \ldots, \mathrm{N}_{\mathrm{b}}$, where $\mathrm{N}_{\mathrm{b}}$ is the number of divided blocks.

\subsubsection{Gap Bins Location}

The zero-height gap bins is found as in Section B. The position of detected gap bins is labeled as

$\mathrm{V}_{\mathrm{g}}{ }^{\mathrm{i}}=\left[\mathrm{V}_{\mathrm{g}}{ }^{\mathrm{i}}(0), \mathrm{V}_{\mathrm{g}}{ }^{\mathrm{i}}(1), . ., \mathrm{V}_{\mathrm{g}}{ }^{\mathrm{i}}(\mathrm{k}), \ldots, \mathrm{V}_{\mathrm{g}}{ }^{\mathrm{i}}(255)\right]$

where $\mathrm{V}_{\mathrm{g}} \mathrm{i}(\mathrm{k})=1$, if the bin at $\mathrm{k}$ is a gap orelse $\mathrm{V}_{\mathrm{g}}{ }^{\mathrm{i}}(\mathrm{k})=0$.

\subsubsection{Peak Bins Location}

Peak bins which are like impulse noise can be located by median filtering. The gap bins are first filled with the average of neighboring bins, then median filtering is applied to the gap-filled histogram. Then peak positions are located by thresholding the difference between the gap-filled histogram and the filtered version. It can be seen that peak bins are not detected in the primary image. The detected peak positions is as $V_{P}{ }^{i}=\left[V_{P}{ }^{i}(0), V_{P}{ }^{i}(1), \ldots \ldots, V_{P}{ }^{i}(k), \ldots, V_{P}{ }^{i}(255)\right]$ where $\mathrm{V}_{\mathrm{P}}{ }^{\mathrm{i}}(\mathrm{k})=1$ refers to a peak.

To further decrease detection errors, we apply a simple and strict threshold-based binarization to

$$
C_{g}=\sum_{\mathrm{i}=1}^{\mathrm{Nb}} \mathrm{V}_{\mathrm{gi}} / \mathrm{N}_{\mathrm{b}}
$$

The detected co-existing gap positions are recorded as $\mathrm{Vg}=$ $[\operatorname{Vg}(0), \operatorname{Vg}(1), \ldots, \operatorname{Vg}(\mathrm{k}), \ldots, \operatorname{Vg}(255)]$, where $\operatorname{Vg}(\mathrm{k})=1$, if $\mathrm{Cg}(\mathrm{k})$ is larger than the threshold; $\operatorname{Vg}(\mathrm{k})=0$, otherwise. The corrected gap position vector, $\mathrm{V}_{\mathrm{gc}} \mathrm{i}$, is generated as in (6)

$$
\mathrm{V}_{\mathrm{gc}}{ }^{\mathrm{i}}=\mathrm{V}_{\mathrm{g}}{ }^{\mathrm{i}} \odot \mathrm{V}_{\mathrm{g}}
$$

where $\odot$ denotes Hadamard product. Similarly, the corrected peak position vector $\mathrm{V}_{\mathrm{pc}}{ }^{\mathrm{i}}$ can also be obtained.

\subsubsection{Gap Based Similarity Measure}

To differentiate between two source regions, we should first set a reference position vector. Then each block can be classified by the similarity between the position vector and the reference one. The reference gap position vector is given as $\mathrm{V}_{\mathrm{gr}}=\mathrm{V}_{\mathrm{gc}}{ }^{\mathrm{k}}$, where

$$
\mathrm{k}=\operatorname{argmax}_{\mathrm{ie}\left\{1_{1}, 2 \mathrm{~N} \mathrm{~N}_{\mathrm{b}}\right\}}\left(\left|\mathrm{V}_{\mathrm{gc}}\right|\right)
$$

To measure the similarity between the gap position vectors $\mathrm{V}_{\mathrm{gc}}{ }^{\mathrm{i}}$ and $\mathrm{V}_{\mathrm{gr}}$, each gap-involved pair $\mathrm{V}_{\mathrm{gc}}{ }^{\mathrm{i}}$ and $\mathrm{V}_{\mathrm{gr}}(\mathrm{k})$ should be investigated. As in Fig. 5, there exist three possible correspondences for a gap-involved pair. They are given in (8)

$$
\begin{aligned}
& \text { (1) } \mathrm{V}_{\mathrm{gr}}(\mathrm{k})=1, \mathrm{~V}_{\mathrm{gc}}{ }^{\mathrm{i}}(\mathrm{k})=1 \text {; } \\
& \text { (2) } \mathrm{V}_{\mathrm{gr}}(\mathrm{k})=0, \mathrm{~V}_{\mathrm{gc}} \mathrm{i}(\mathrm{k})=1 ; \\
& \text { (3) } \mathrm{V}_{\mathrm{gr}}(\mathrm{k})=1, \mathrm{~V}_{\mathrm{gc}}{ }^{\mathrm{i}}(\mathrm{k})=0 \text {; }
\end{aligned}
$$

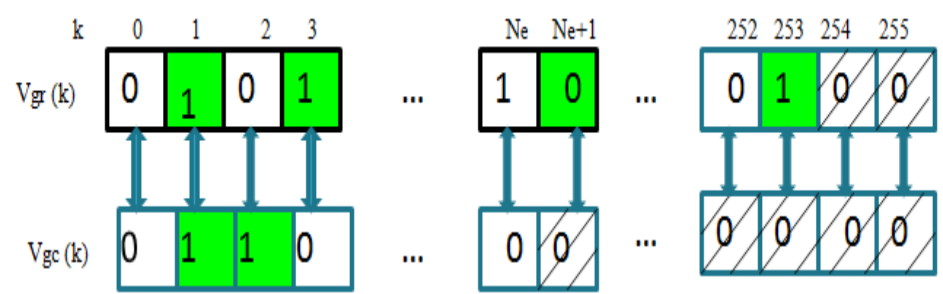

Figure 5: Correspondence between $\mathrm{V}_{\mathrm{gr}}(\mathrm{k})$ and $\mathrm{V}_{\mathrm{gc}}$

The pair is completely matched in the case (1). The more pairs occur as such, the more similar the two gap position distributions. In case (2), the detected gap is not marked in the reference vector. In the case (3), the gap in the reference vector is absent in the unlabeled histogram. Both (2) and (3) attribute to the different contrast enhancement mapping applied to the image region out of the reference block. The 


\section{International Journal of Science and Research (IJSR) \\ ISSN (Online): 2319-7064}

Index Copernicus Value (2013): 6.14 | Impact Factor (2014): 5.611

similarity between $\mathrm{V}_{\mathrm{gc}}{ }^{\mathrm{i}}$ and $\mathrm{V}_{\mathrm{gr}}$, denoted by $\mathrm{m}_{\mathrm{g}}^{\mathrm{i}}$, can be defined as the (9)

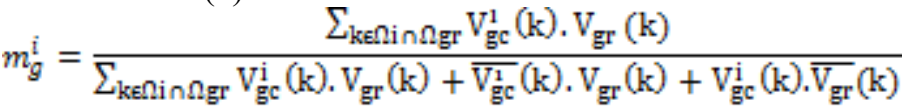

$\bar{V}$ is the element-wise complement of $\mathrm{V}$, that is,

$\bar{V}(\mathrm{k})=1-\mathrm{V}(\mathrm{k}), \mathrm{k}=0,1,2, \ldots, 255 . \Omega_{\mathrm{f}} \cap \Omega_{\mathrm{gr}} \quad$ denotes the intersection between EDR's of $\mathrm{V}_{\mathrm{gc}} \mathrm{i}$ and $\mathrm{V}_{\mathrm{gr}}$. When no gapinvolved pair is detected in the EDR(Effective Detection Range) intersection, we mark $\mathrm{m}_{\mathrm{g}}{ }^{\mathrm{i}}=-1 . \mathrm{m}_{\mathrm{g}}{ }^{\mathrm{i}}$ becomes larger if more gaps co-exist. $\mathrm{m}_{\mathrm{g}}{ }^{\mathrm{i}}$ approaches the maximum 1 if all gaps are matched, and decreases to 0 if all gap-involved pairs behave as (2) (3).

\subsubsection{Peak Based Similarity Measure}

Since the image block usually owns a narrow histogram, the gap bins might be unavailable in a few blocks. The narrow histogram without gap bins might carry with peak bins. Specifically, we have in (10)

$$
\mathrm{V}_{\mathrm{pr}}(\mathrm{k})=\ell\left(\sum_{\mathrm{neN}} V_{p e}^{\mathrm{n}}(k)>0\right)
$$

Here, $\mathrm{k}=0,1,2, \ldots, 255 . \ell(\cdot)$ is the indicator function, $\mathrm{N}_{\mathrm{R}}=$ \{i $\left.\mid \mathrm{m}_{\mathrm{g}}{ }^{\mathrm{i}}>\mathrm{t}_{\mathrm{g}}\right\}$, where $\mathrm{t}_{\mathrm{g}}$ is the threshold. As $\mathrm{m}_{\mathrm{g}}{ }^{\mathrm{i}}$ defined in (9), the similarity between $\mathrm{V}_{\mathrm{pc}}{ }^{\mathrm{i}}$ and $\mathrm{V}_{\mathrm{pr}}$, marked as $\mathrm{m}_{\mathrm{p}}{ }^{\mathrm{i}}$, is defined in the same form by replacing the gap variables with the corresponding peak ones. If no peak involved pair exists in EDR intersection, we mark $m_{p}{ }^{i}=-1$.

\subsubsection{Similarity Maps Fusion For Composition Detection}

The blockwise similarities $\mathrm{m}_{\mathrm{g}}{ }^{\mathrm{i}}=-1$ or $\mathrm{m}_{\mathrm{p}}{ }^{\mathrm{i}}=-1$ are updated by averaging the neighboring measurements. The similarity for the i-th unlabeled block, denoted by $\mathrm{m}^{\mathrm{i}}$, can be generated by fusing the peak/gap based similarities. When $\mathrm{m}_{\mathrm{g}}{ }^{\mathrm{i}}=-1$ and $\mathrm{m}_{\mathrm{p}}{ }^{\mathrm{i}}=-1$, we compute as in (11)

$$
\begin{gathered}
\mathrm{m}^{\mathrm{i}}=\left(\mathrm{m}_{\mathrm{g}}^{\mathrm{i}}+\mathrm{m}_{\mathrm{p}}^{\mathrm{i}}\right) / 2 \\
\text { when } \mathrm{m}_{\mathrm{g}}^{\mathrm{i}}=-1 \text { or } \mathrm{m}_{\mathrm{p}}^{\mathrm{i}}=-1, \mathrm{~m}^{\mathrm{i}}=\max \left(\mathrm{m}_{\mathrm{g}}^{\mathrm{i}} \mathrm{m}_{\mathrm{p}}^{\mathrm{i}}\right) \text {. }
\end{gathered}
$$

\section{Simulation Results and Discussions}

Unaltered photograph images is used in raw format. Natural photographs, uncompressed images on various topics such as natural scenes, man-made objects, indoors and outdoors captured by several different cameras, stored in JPEG format and with size from $1200 \times 900$ to $2832 \times 2128$ pixels. All camera settings including exposure and white balance are set automatic for resembling what probably common users would do. The Figures 6-8 shows the various output images during running the program. The receiver operating characteristic (ROC) curves are generated for evaluation.

\subsection{Identifying Source- Enhanced Composite Images}

The images are tested to know whether it is source enhanced or not and the result is displayed.

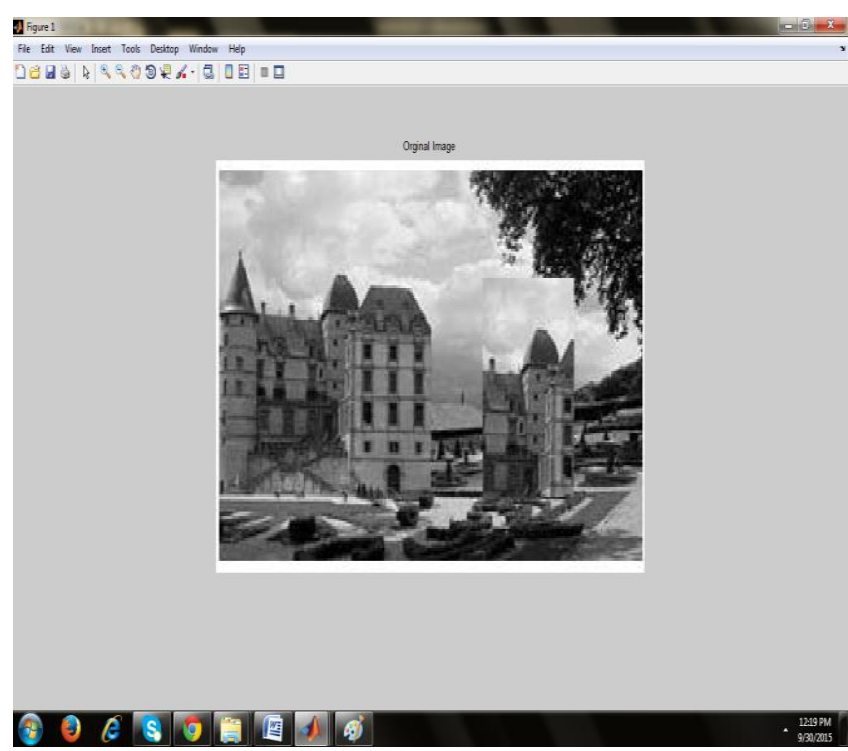

Figure 6: Input Image

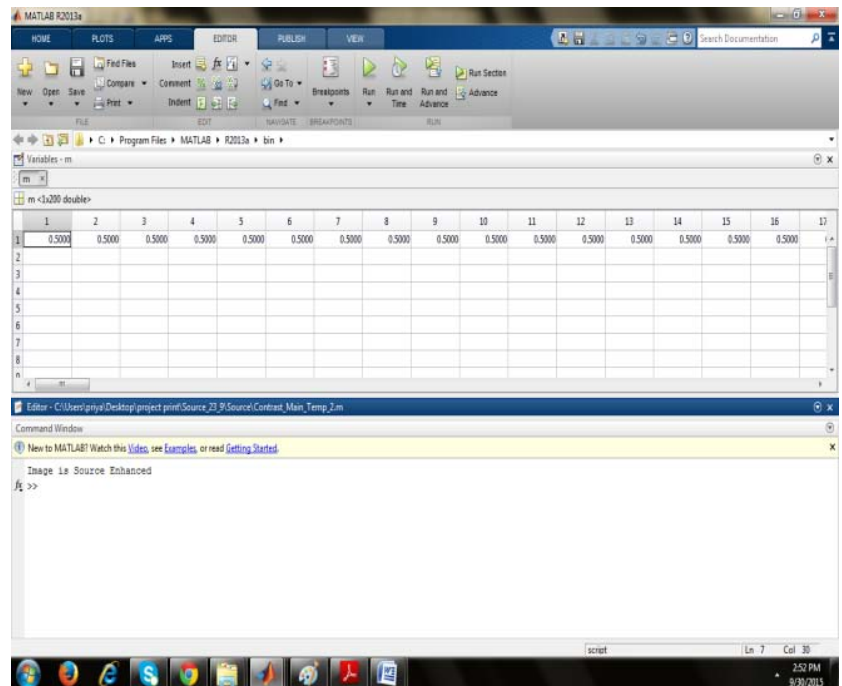

Figure 7: By similarity map fusion showing an image is source enhanced

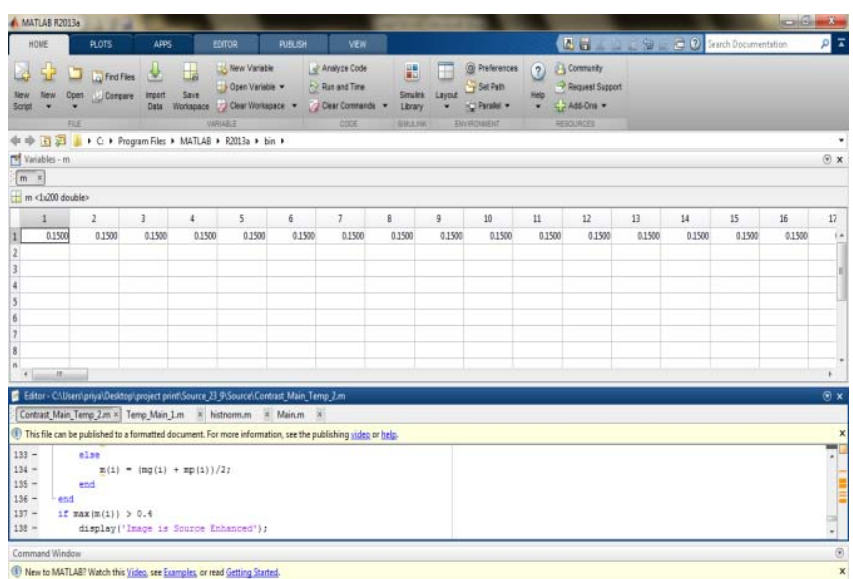

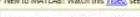

OEs

Figure 8: By similarity map fusion showing an image is not source enhanced 


\section{Conclusoin}

Here proposed two contrast enhancement based forensic algorithms based on histogram peak/gap artifacts analysis. First, extended to detect the global contrast enhancement in both uncompressed and already JPEG-compressed images. The histogram peak/gap artifacts incurred by the JPEG compression and pixel value mappings are analyzed theoretically. A regular pixel value mapping relationship exists in flat regions, but not in other regions. Therefore, the zero-height gap feature can be used to detect global contrast enhancement in both uncompressed and compressed images. Second, proposed a new method to find out both source enhanced composite image, which was not validated in the previous forensics methods. Positional information could serve as fingerprinting feature for identifying different contrast enhancement manipulations. Consistency between the peak/gap artifacts detected in different regions is checked for discovering composite images.

\section{References}

[1] Gang Cao, Yao Zhao, Rongrong Ni and Xuelong Li "Contrast Enhancement-Based Forensics in Digital Images," IEEE Transactions on information forensics and security, vol. 9, no. 3, March 2014A.

[2] H. Farid, "Image forgery detection," IEEE Signal Process. Mag., vol. 26, no. 2, pp. 16-25, Mar. 2009

[3] B. Mahdian and S. Saic, "A bibliography on blind methods for identifying image forgery," Image Commun., vol. 25, no. 6, pp. 389-399, Jul. 2010.

[4] S. Bayram, I. Avcubas, B. Sankur, and N. Memon, "Image manipulation detection," J. Electron. Imag., vol. 15, no. 4, pp. 04110201-04110217, 2006.

[5] A. Swaminathan, M. Wu, and K. J. R. Liu, "Digital image forensics via intrinsic fingerprints," IEEE Trans. Inf. Forensics Security, vol. 3, no. 1, pp. 101-117, Mar. 2008.

[6] G. Cao, Y. Zhao, R. Ni, and H. Tian, "Anti-forensics of contrast enhancement in digital images," in Proc. ACM Multimedia Security Workshop, Rome, Italy, 2010, pp. 25-34.

\section{Author Profile}

Priya Ann Koshy, BE in Electronics and Communication from SUN College of Engineering and Technology, Anna University. Currently doing Mtech in Applied electronics in M.G University College of Engineering.

Kavitha .N. Nair, Lecturer Dept. of ECE, University college of Engineering, Thodupuzha, Kerala. 